\title{
3D Printing of Functional Anatomical Insoles
}

\author{
Miguel Davia-Aracil ${ }^{1,2}$ • Juan José Hinojo-Pérez ${ }^{1}$ • Antonio Jimeno-Morenilla2* Higinio Mora-Mora ${ }^{2}$
}

\begin{abstract}
Anatomical insoles and additions have a corrective action on the footwear user. They are intended to reduce and adequately distribute plantar pressure among support points, thus minimising the stress these points can undergo. Such customised components have traditionally been manufactured by subtractive techniques, i.e. by milling a sheet of material. Latest advances in additive manufacturing (AM) techniques and, in particular, the popularisation of 3D printing by fused deposition modelling (FDM), have opened new ways for the production of anatomical insoles. These technologies allow additional functionalities to be added, as for instance the use of materials with antimicrobial properties, or, at a structural level, zonal control in 3D design to increase cushioning capacity. The latter cannot be achieved by traditional manufacturing techniques, in that the inside of the element is not accessible. However, there are no CAD tools available for the design and production of insoles, which are specifically oriented to take advantage of the benefits that AM can bring about. This paper describes a new methodology intended for the functionalisation of anatomical insoles through a systematic approach. On the one hand, internal structures are automatically obtained by parametric design; on the other hand, the 3D geometry of the insole or addition is adequately processed so that it can be printed by FDM, thus circumventing the constraints of this production technique.
\end{abstract}

Keywords Anatomical insoles - Customisation - Additive manufacturing - Functionalisation - Cushioning

\footnotetext{
* Corresponding author's email: jimeno@dtic.ua.es

1 Spanish Footwear Technology Institute (INESCOP), Polígono

Campo Alto, 03600 Elda, Spain

2 Department of Computer Technology, University of AlicanteCtra,

San Vicente del Raspeig S/N, 03690 Alicante, Spain
}

\section{Acknowledgements}

This paper has been prepared and published in the framework of the research project AMFAB II - Fabricación avanzada de productos manufactureros tradicionales mediante tecnologías de Additive Manufacturing (IMDECA/2016/2). The authors thank the Valencian Institute for Business Competitiveness (IVACE) and the FEDER European Union funds for their financial support to this project.

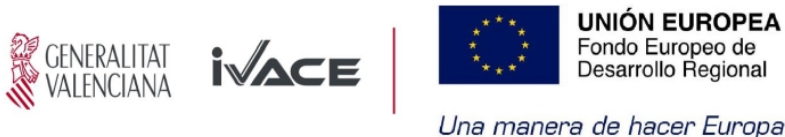




\section{D Printing of Functional Anatomical Insoles}

\begin{abstract}
Anatomical insoles and additions have a corrective action on the footwear user. They are intended to reduce and adequately distribute plantar pressure among support points, thus minimising the stress these points can undergo. Such customised components have traditionally been manufactured by subtractive techniques, i.e. by milling a sheet of material. Latest advances in additive manufacturing (AM) techniques and, in particular, the popularisation of 3D printing by fused deposition modelling (FDM), have opened new ways for the production of anatomical insoles. These technologies allow additional functionalities to be added, as for instance the use of materials with antimicrobial properties, or, at a structural level, zonal control in 3D design to increase cushioning capacity. The latter cannot be achieved by traditional manufacturing techniques, in that the inside of the element is not accessible. However, there are no CAD tools available for the design and production of insoles, which are specifically oriented to take advantage of the benefits that AM can bring about. This paper describes a new methodology intended for the functionalisation of anatomical insoles through a systematic approach. On the one hand, internal structures are automatically obtained by parametric design; on the other hand, the $3 \mathrm{D}$ geometry of the insole or addition is adequately processed so that it can be printed by FDM, thus circumventing the constraints of this production technique.
\end{abstract}

Keywords Anatomical insoles - Customisation - Additive manufacturing - Functionalisation - Cushioning

\section{Introduction}

In the field of footwear, the insole (see Figure 1) is a component located between the foot and the shoe sole. Being in direct contact with the foot [1], the insole supports body weight. Therefore, it directly affects the biomechanics of the foot and the body as a whole [2]. An anatomical insole should be intended to reduce and adequately distribute plantar pressure among support points, thus minimising the stress these points can undergo during the execution of physical activity or while standing for long periods. Incorrect support may lead to injuries in the long term. This is even worse when wearers suffer from certain illnesses, such as diabetes, where correct pressure distribution is critical to prevent ulceration [3]. There are certain essential aspects characterising an insole. Firstly, its morphology, in that it has to be adapted to the anatomy of the foot sole, which is characterised by three main arches. These arches shall be adequately balanced to achieve perfect support. Secondly, the density of the material(s) chosen for production, which directly redounds to certain properties, like flexibility, hardness, resistance, shock absorption, etc. There are also other corrective elements, called additions (see Figure 2), which are added on the metatarsal area of the insole in order to retain the foot in an anatomically correct position and dampen impacts while walking. These are common elements in high-heeled shoes, where the metatarsal area supports greater weight than other areas of the foot.

Traditionally, the production of anatomical insoles for a given user was carried out by subtractive manufacturing (SM) techniques. This is a process by which objects are constructed by successively cutting material away from a solid block of material using a $\mathrm{CNC}$ machine. With regard to the production of anatomical insoles, subtractive manufacturing poses certain drawbacks [4]. First of all, material waste upon cutting material away from the original block. Secondly, lack of flexibility to alternate materials in different areas according to the user's needs, since the material to be used is pre-determined by the original block. Thirdly, it is impossible to gain access to the inside of the insole. Nevertheless, the recent advances in additive manufacturing allow the limitations inherent to SM to be overcome. 


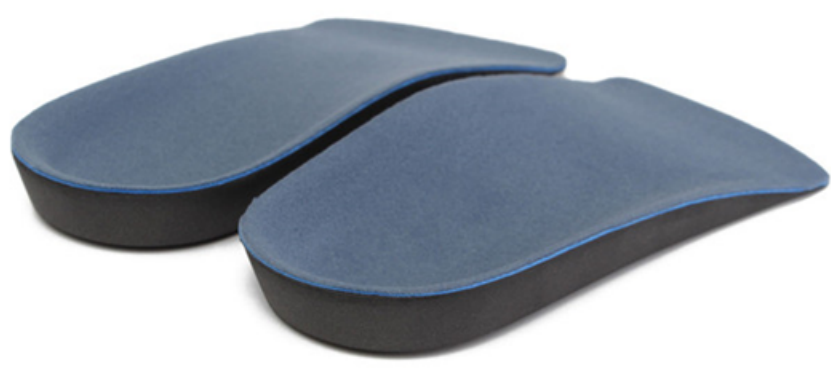

Fig. 1. Anatomic insole.

Additive manufacturing (AM) is a process by which a three-dimensional object is built up by superimposing material layers using different manufacturing techniques. Although additive manufacturing dates back to 1987, it has gained momentum over recent years thanks to the advances in technology and cost reduction, which allows very complex objects to be manufactured in short time and at a competitive cost. There are different additive manufacturing techniques, such as Fused Deposition Modelling (FDM), Polyjet or Selective Laser Melting (SLM) [5]. As far as anatomical insole production is concerned, this paper focuses on additive manufacturing by FDM using $3 \mathrm{D}$ printers, where a nozzle moving over a plane melts a plastic filament. The reasons for this is the low cost of the equipment, which makes it suitable for use by the light industry, as is the case of the footwear sector, and the fact that 3D printing systems can add different polymeric materials to the object to be produced.

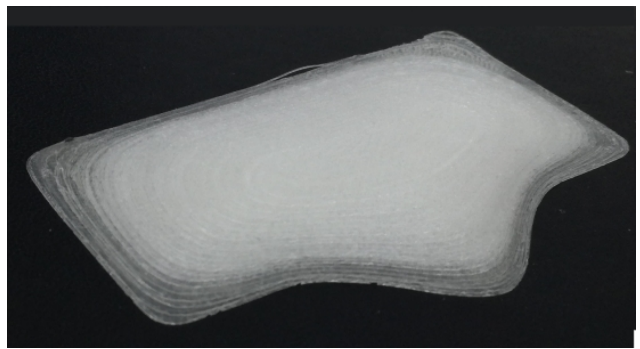

Fig. 2. Insole addition.
The use of FDM 3D printing for the manufacture of anatomical insoles also brings about new production possibilities which would otherwise not be feasible by traditional methods. Among these, the possibility to add new properties to the insole stands out; this way, new specific functionalities are conferred on the insole, which provide added value. This is called functionalisation. On the one hand, it is possible to functionalise the material by adding an extra component that provides new features. For instance, it is possible to create a filament to be extruded that incorporates an antimicrobial compound to prevent the onset of infections [6]. On the other hand, manufacturing by FDM allows the functionalisation of the geometric structure of insoles by incorporating internal elements by zones. Such elements are to modify the intrinsic properties of the material employed as well as the external geometry of the insole. For example, hollow areas can be defined in the insole design, which will modify the insole functionality in terms of shock absorption, flexibility, etc.
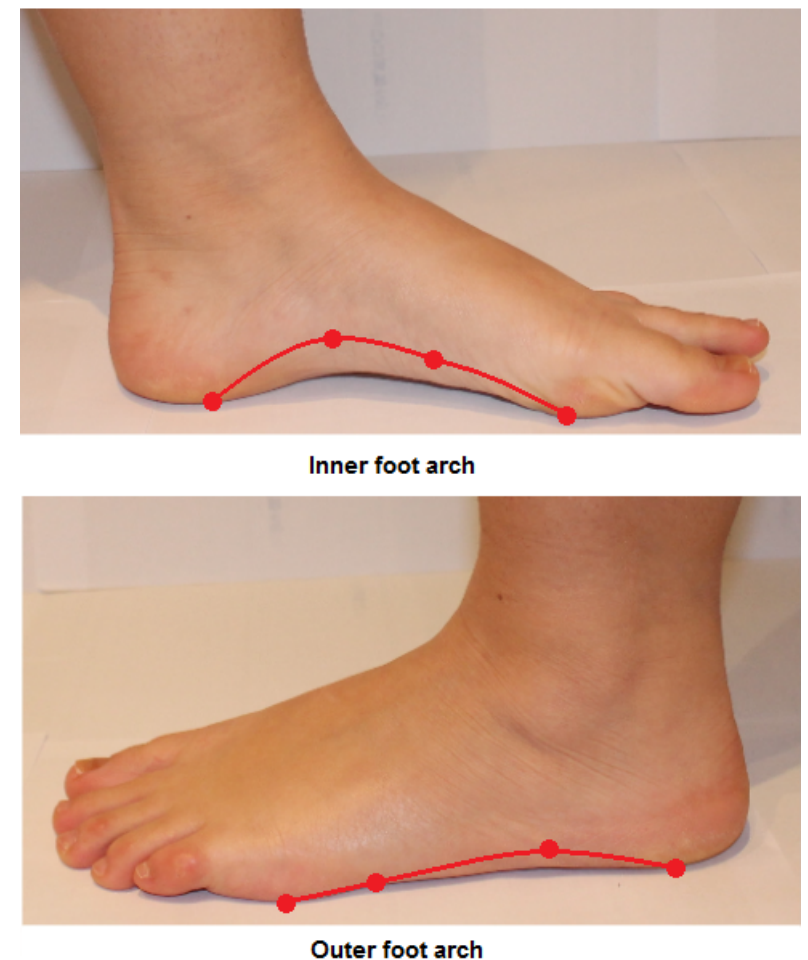

Fig. 3. Inner and outer foot sole arches.

Therefore, additive manufacturing alleviates many of the constraints derived from the use of the Design for Manufacturing (DFM) paradigm [7]. Under this paradigm, the designer is to create the design always taking into account the limitations established by the manufacturing system. Although there is significant improvement with respect to traditional manufacturing, none of the $3 \mathrm{D}$ printing systems is completely free from limitations. This becomes evident in the above-described example on the production of 
anatomical insoles with functionalised structure by FDM. In this case, the main software applications for printing, like Ultimaker Cura, pose the drawback of managing the insole geometry as a water-tight object and ignore any structure contained therein, in such a way that the insole is manufactured as a completely solid object. It is therefore necessary to create a specific CAD tool for insoles that makes it possible to provide the 3D model with the relevant properties that allow precise manufacture by FDM.

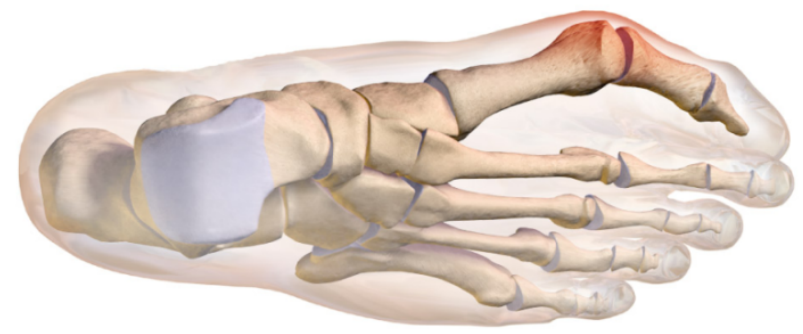

Fig. 4. Hallux Valgus is a deformity on the first metatarsophalangeal joint.

Based on a previous study about the possibilities for material and structural functionalisation of anatomical insoles, this paper intends to propose several CAD methodologies aimed at the design and production of insoles by additive manufacturing, more specifically, the design of internal structures that can alter the shock absorption capacity in different areas, and the processing of the geometry prior to FDM 3D printing.

This paper is structured as follows: Section 2 includes a state-of-the-art review on anatomical insole production technologies, materials, functionalisation of polymeric materials and 3D printing. Section 3 describes a new methodology specifically developed for insole design and production based on AM. Section 4 presents several experiments aimed at checking how the addition of internal structures modifies the insole's shock absorption capacity. Finally, the study conclusions are presented.

\section{Research background}

This section presents a study of the essential issues related to the proposed methodology. First of all, the fundamentals of anatomical insoles and their benefits are examined. Section 2.2 describes the most commonly used materials for insole production. Section 2.3 describes the process for the functionalisation of materials and its usefulness. Section 2.4 reviews the state-of-the-art of additive manufacturing, focusing on FDM 3D printing. Finally, parametric design fundamentals are explained.

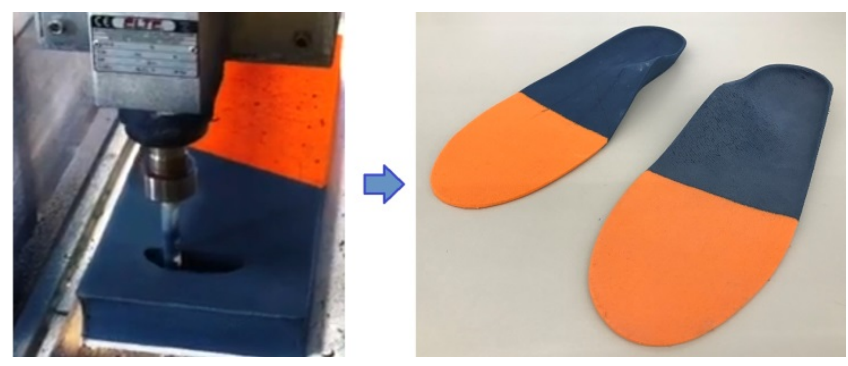

Fig. 5. Two-density EVA sheets are milled for the production of anatomical insoles

\subsection{Anatomical insoles.}

The foot comprises 26 bones, 33 joints and over 100 muscles, tendons and ligaments, which make up a complex structure that remains in fine equilibrium [1]. The foot sole has three main arches. First of all, there is the medial longitudinal arch, which goes between the calcaneus and the first metatarsal head; secondly, there is the lateral longitudinal arch in contact with the ground; and finally, the transverse arch is located between the first and fifth metatarsal heads. These arches, as shown in Figure 3, have to be adequately balanced to achieve perfect support of the foot while walking and standing.

Unbalance in the forces acting on foot arches is provoked by the weakening of ligaments supporting the plantar fascia, and is the cause of biomechanical disorders, such as pes planus and pes cavus. Pes planus is characterised by the fact that plantar arches are partially or completely fallen. According to Cacace et al. [8], pes planus is estimated to affect approximately $3-25 \%$ of the adult population globally. Although in most cases this condition does not cause any pain, people suffering this problem have a higher odds of developing changes in bone structures in the long term, such as Hallux Valgus or bunion (see Figure 4) and Hallux Rigidus (osteoarthritis of the big toe joint). Pes cavus is a type of foot with an excess arch in the plantar fascia, i.e. in pes cavus the contact surface in the midfoot and toe areas of the foot sole are reduced and consequently pressure on the rest of the foot areas resting on the ground increases. According to [9], although this condition affects a smaller 
population percentage than pes planus $(10-15 \%), 60 \%$ of these are likely to suffer pain and associated pathologies, including calluses, changes in bone structures, limited joint mobility, and arterial disease.

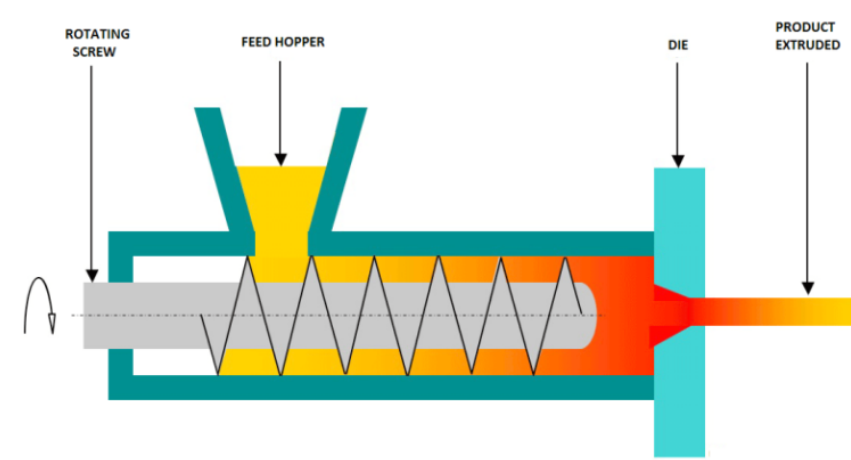

Fig. 6. Extrusion process of polymeric pellets into a filament..

Thanks to the use of anatomical insoles, it is possible to dampen impacts while walking, as well as to reduce and redistribute plantar pressure adequately. [10] presented a study investigating the effects of anatomical insoles through finite element analysis of 3D models of the foot together with insoles. The results obtained showed that pressure was reduced in most of the plantar regions, the reduction ratios ranging from $19 \%$ to $56 \%$.

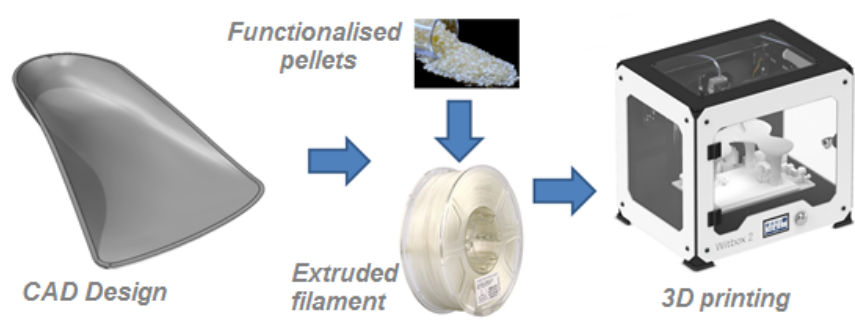

Fig. 7. 3D printing allows the manufacture of a CAD design from functionalised polymeric pellets.

As a complement to the anatomical insole, it is possible to add certain corrective elements, called additions. Such elements are located underneath the metatarsal area, and are commonly used in high-heeled shoes, where this area withstands greater pressure than other areas on the foot. Their main function is to alleviate pressure in this area by dampening impacts while walking and retaining the foot in an anatomically correct position.

\subsection{Materials used for insole production.}

A wide range of materials have traditionally been employed for the production of shoe insoles. Properties such as hardness, tensile strength, rigidity, flexibility and permeability directly depend on the constituent material and have been the object of some studies $[11,12]$. One of the most commonly employed materials over recent years is ethylene-vinyl acetate or EVA, which is a thermoset polymer supplied in sheet form in different thicknesses and sizes. [13] compared hardness and shock absorption properties of insoles made from three EVA foams of different density. A usual solution for the production of anatomical insoles is milling EVA blocks with different density areas using a three-axis CNC machine, as shown in Figure 5. The highest density area is used to produce the rear part of the insole, as it is characterised by higher hardness and provides more stability to the heel area. The least hard area is used to mill the forepart, since the metatarsal area calls for higher impact absorption efficiency [14]. As can be observed, this solution, even though it is more suitable for production using a single material, is little flexible and very limited. The possibility of functionalising anatomical insoles by providing them with internal structures that locally modify shock absorption properties could meet user's needs in terms of comfort, pressure distribution correction and impact absorption in a more extensive and flexible way than how it has been done till present. However, this is not possible using traditional subtractive manufacturing techniques.

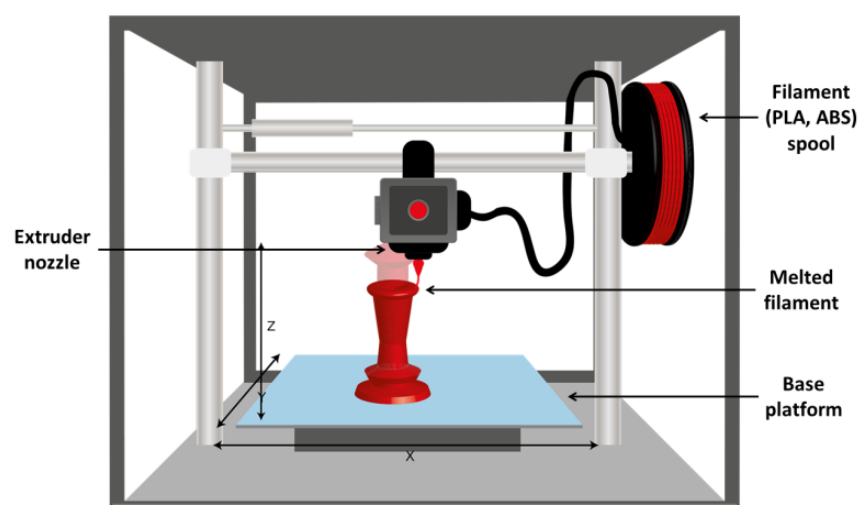

Fig. 8. Elements making up a FDM 3D printer 


\subsection{Functionalised materials}

A functionalised material is a material that combines two or more properties where only the first one is structural, i.e. is inherent to the material itself, and the rest of the properties have been provided by means of additivation using different particles, such as antimicrobial, electroluminescent, thermochromic or conductive additives, which allow the functionalised material to meet an auxiliary need. With regard to wearable products, like footwear, the most interesting properties are those related to health, since antimicrobial [6], antibacterial or controlled drug release properties can be conferred on the product.

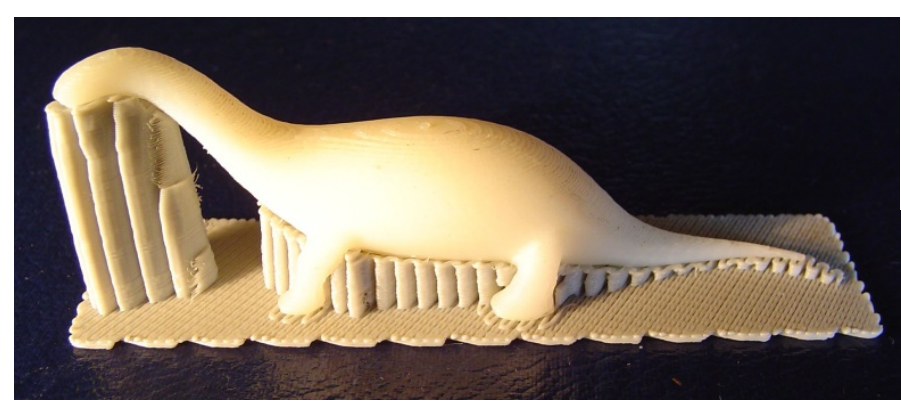

Fig. 9. Where the object to be manufactured does not have a support base, a support made from a water-soluble based material is added.

One of the main advantages of FDM 3D printing is that it allows thermoplastic polymers to be used [15], such as polylactic acid (PLA) [16] or acrylonitrile butadiene styrene (ABS) [17], which have been previously functionalised. The process to obtain this type of materials starts by adding an additive with certain properties to the polymeric pellets. Then, the filament is produced, which will serve as the base for 3D printing. As shown in Figure 6, pellets are fed into the machine, where they are melted by a rotating spindle at a controlled temperature, and the resulting product goes through a nozzle that lends the characteristic filament shape.

With regard to insole production, the filament used for FDM printing can be functionalised to meet purposes other than its original function of balancing pressures and attenuating impacts. This is achieved by conferring antimicrobial properties on the original material (Figure 7) with the aim of avoiding microorganism proliferation, which apart from aesthetical issues, like bad odour, can also have health effects. [18] proposed two general strategies to produce antimicrobial resins to be used as a base material for 3D printing. [19] investigated the use of polymer blends able to release drugs in a controlled and adjustable way that are printable by FDM.
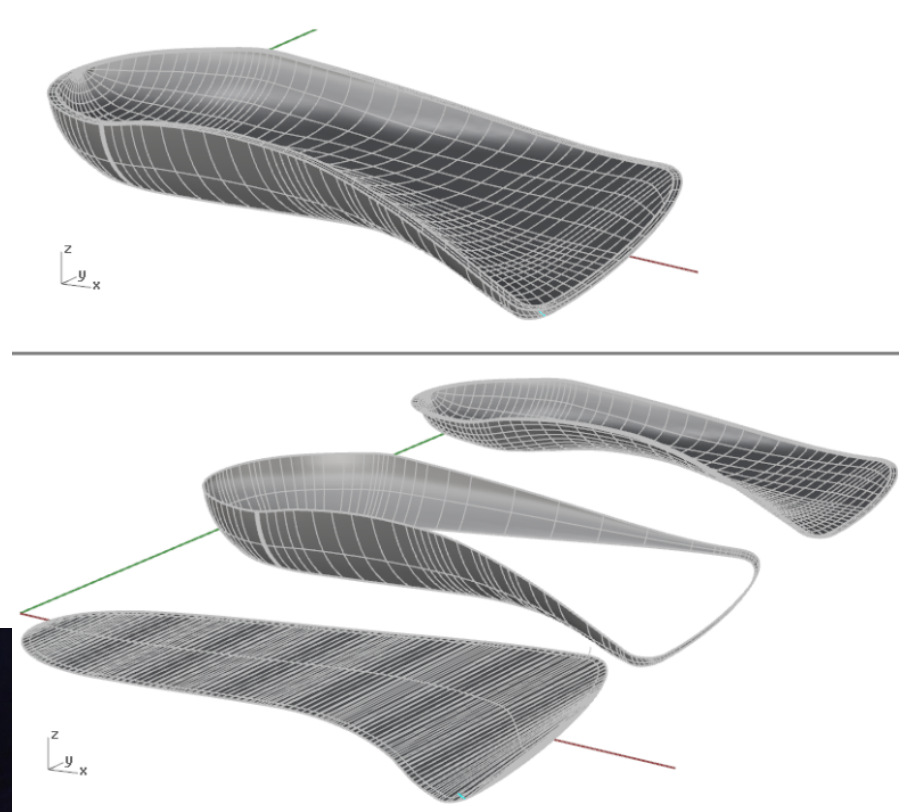

Fig. 10. Top: 3D insole in the described position. Bottom: surfaces making up the insole displayed separately.

\subsection{Additive manufacturing -FDM printing.}

Additive manufacturing is defined by a range of technologies that are capable of translating virtual solid model data into physical models in a quick and easy process by adding material. The physical object is obtained through a process consisting in depositing successive layers of material of a finite thickness from bottom to top. This methodology, the technology of which has witnessed substantial improvements in recent years, brings about new possibilities when compared with traditional systems, thus avoiding many constraints. The unique capabilities of AM [20] include: Shape complexity: it is possible to build virtually any shape avoiding the approximations imposed by subtractive or machining methods.; b) Material complexity: as illustrated in section 2.3, it is possible to use material combinations to provide the product with new properties; c) Hierarchical complexity: multi-scale hierarchical structures can be designed and fabricated from the microstructure (size in the millimetre range) to macrostructure; d) Functional complexity: different geometries can be embedded in an object in such a way that the resulting product features new or different functionalities. These benefits can help AM notably increase industrial competitiveness in that it ensures quick responsiveness to changing market needs and growing consumer demands for product customisation [21].

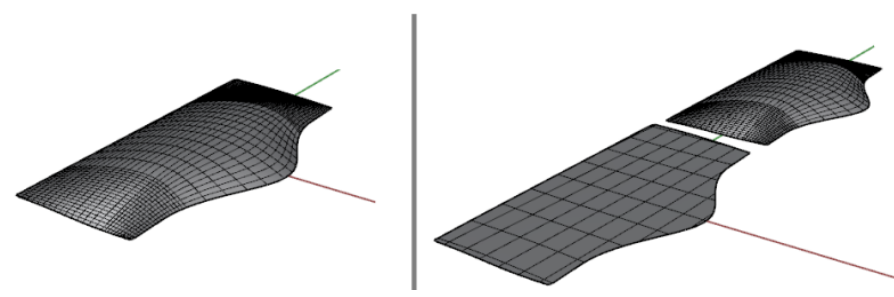


Fig. 11. 3D addition and the surfaces that make it up.

The advantages of AM lead to the reconsideration of the DFM (Design For Manufacturing) paradigm, where design is conditional, on the one hand, on limitations when it comes to manufacturing the object, and, on the other hand, on manufacturing parameters. The latter can be related to the type of tool to be used, the tool penetration angle, or the angle for part removal from the mould. However, although AM brings about new possibilities in terms of geometry and materials with respect to traditional methods, it is not free from certain limitations. [22] explained that there are two main constraints. Firstly, the nozzle stays parallel to the vertical axis, so this leads to accessibility constraints to avoid collisions between the nozzle and the part. Secondly, acceleration or deceleration of the nozzle can cause variations of height of the material deposited and thus this should be minimised by smoothing sharp corners in the design.
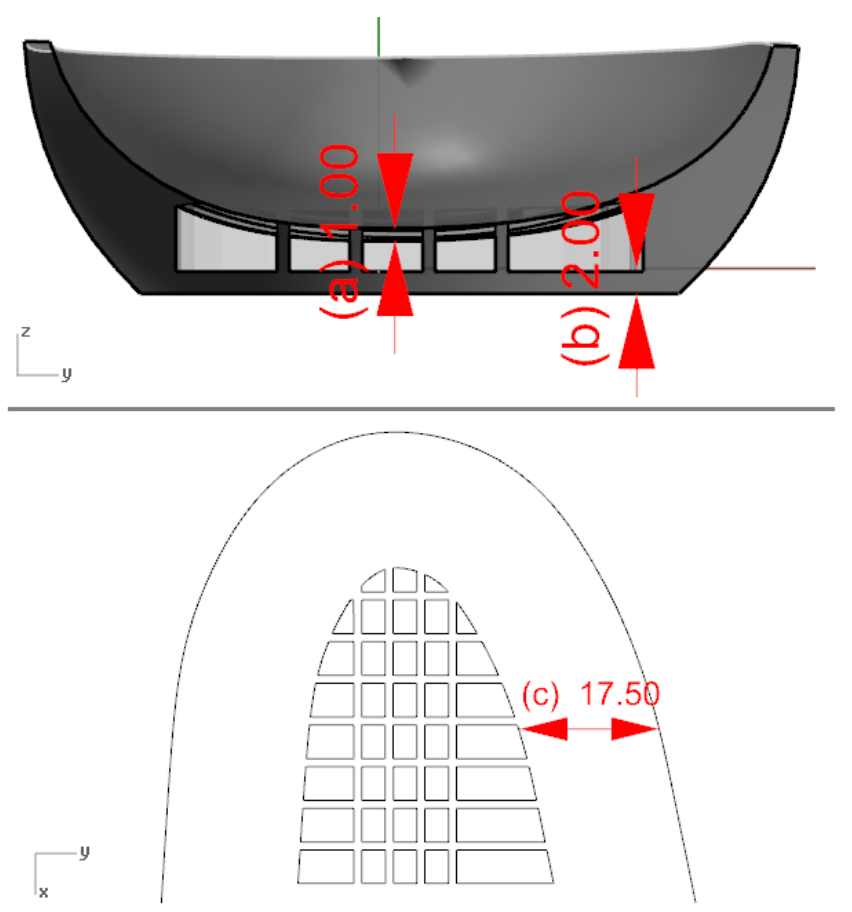

Fig. 12. Top: Lateral insole section where upper (a) and lower (b) offset are depicted. Bottom: Foot sole curve and structure projection on $\mathrm{Z}$ plane as well as foot sole offset (c).
It is therefore necessary to adapt traditional design methodologies to additive manufacturing through the DFAM (Design For Additive Manufacturing) paradigm. [7] defined DFAM as a set of shapes, sizes, material compounds, geometric mesostructures and microstructures to take full advantages of the possibilities of this production method, in such a way that the original design is implemented to reduce costs and produce objects that were unfeasible before.

\begin{tabular}{|c|c|}
\hline \multicolumn{2}{|l|}{ Insole structures } \\
\hline Insole structure & $1++-$ \\
\hline Base surface & Surface \\
\hline Profile curve & Curve \\
\hline \multicolumn{2}{|l|}{ Units } \\
\hline Unit & $1++-$ \\
\hline Type & Rectangles \\
\hline Angle & $0 \leqslant$ \\
\hline Starting zone & 8 \\
\hline Ending zone &  \\
\hline Offset & $5 \leqslant$ \\
\hline Upper tillet & $0=$ \\
\hline Lower fillet & $0 \div$ \\
\hline \multicolumn{2}{|l|}{ Params } \\
\hline Row distance & $1 \div$ \\
\hline Column distance & $1 \leqslant$ \\
\hline Number of rows & $3 \div$ \\
\hline Number of columns & $10 \div$ \\
\hline
\end{tabular}

Fig. 13. View of the tool for internal structures creation. Parameters regarding position of structures are outlined in red.

One of the most popular additive manufacturing techniques is $3 \mathrm{D}$ printing by Fused Deposition Modelling (FDM). Such printers are capable of creating objects by heating and extruding a thermoplastic filament which is deposited layer upon layer. The wide spreading of this system has been recently boosted by the expiration of the FDM patent and the subsequent worldwide development of low-cost machines by a huge number of companies. [23] proposed a methodology for benchmarking part surface accuracy and finish of three low-cost printers. 
The printing process starts by processing the CAD file using the printing software, adequately positioning it, layering at different heights and calculating the path to be followed by the nozzle. On the one hand, there is the limitation that the majority of software tools, like Ultimaker Cura, manage the object to be produced as a solid water-tight object, without taking into account any hollow structure contained therein when it comes to calculating the layers that would make it up. Usually, the software allows the user to establish the percentage of filling material in order to save material. This implies that the inside of the object may be comprised of a sort of mesh, instead of being completely solid. In any case, the internal structures defined in the design stage are ignored, thus limiting their functional possibilities. The material is melted in droplets that are deposited along the extrusion path. Figure 8 illustrates the main parts of this type of $3 \mathrm{D}$ printer. On the other hand, in FDM the material needs a support base on which it is to be deposited. This is not a problem for objects having a wide base, since the material is deposited from the bottom and the object base is enough to continue adding layers on top of it. However, where the object does not have a support base big enough, then it requires a double-head printer. Such printers extrude the material employed to manufacture the object through the main head, while the other head is used to extrude the watersoluble support material (see Figure 9), which is exclusively used as a scaffolding or support so that the main material can be deposited on it. In the last post-processing stage, the support material is removed from the part by dissolving it in water or other liquid substance [19]. Focusing on the footwear industry, AM is a feasible technology for the manufacture of anatomical insoles, in that customisation of the object to be produced does not increase the cost of the process and the resulting products are completely customised.

However, widespread software solutions for the design of insoles, like AutoDesk OrthoModel, Vorum Canfit or Voxelcare do not include CAD tools specifically oriented to exploiting the benefits of AM. With regard to footwear soles, in [24] a methodology for sole design in line with DFAM objectives was developed. Following this research line, the present paper proposes a new CAD methodology specific for the functionalisation of anatomical insoles through the creation of internal structures and their subsequent fabrication by FDM 3D printing. This new methodology is intended to address the design of structures that would otherwise be unfeasible with traditional manufacturing techniques, while the inherent constraints of FDM printing are circumvented, as is the case of the need for a support base or the disregard of internal elements when the geometry is processed by the printing software.

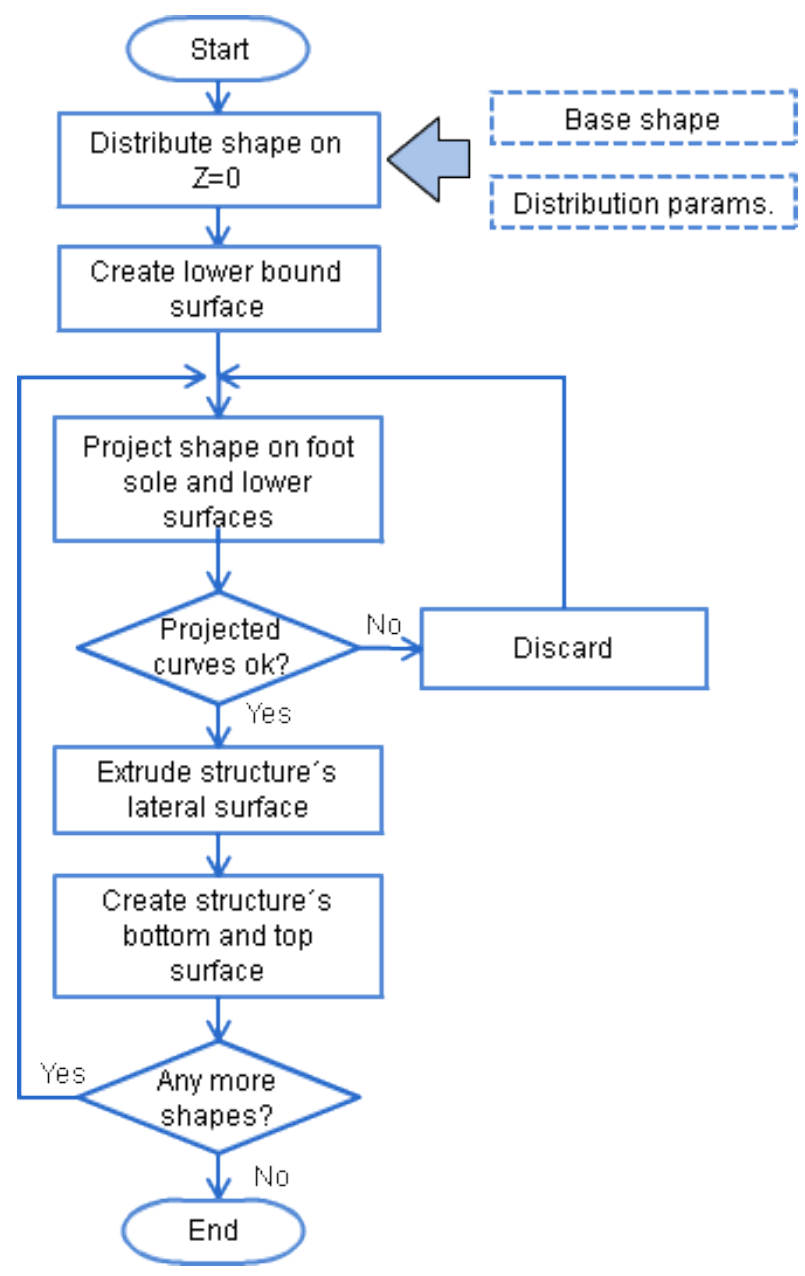

Fig. 14. Data flow diagram for the inner-structure creation algorithm.

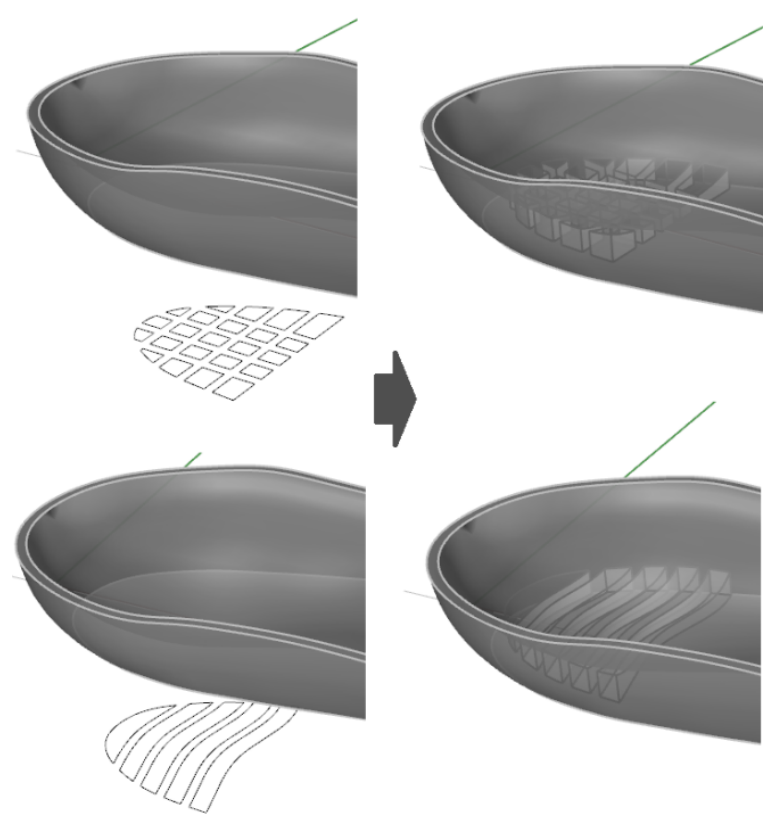

Fig. 15. 2D Pattern and 3D structures obtained from the $2 \mathrm{D}$ pattern, which are then transformed into a functional element in the heel area. 


\subsection{Parametric design}

Parametric design constitutes a paradigm where a relationship between the different elements comprising the object to be modelled is established in the form of a dependence tree [25], which is used to communicate among them the changes in geometry. Such relationships are established as a design component. This implies that whatever change in any of the elements automatically affects all other dependent elements and, this way, structure coherence is automatically maintained. [24] presented a parametric approach to the design of footwear soles. In this line, this paper proposes a parametric methodology for the design of internal structures that are adapted to and dependent on the anatomical insole geometry in an automatic way.

\begin{tabular}{|l|r|}
\hline Type & Rectangles \\
\hline Params & \\
\hline Row distance & \\
\hline Column distance & \\
\hline Number of rows & Diagonal \\
\hline Number of columns & \\
\hline Type & \\
\hline Params & \\
\hline Width & \\
\hline Distance & \\
\hline Diagonal angle & \\
\hline Discard area & Pattern \\
\hline Type & \\
\hline Params & \\
\hline Width & \\
\hline Distance & \\
\hline Type & \\
\hline Params & \\
\hline Diameter & \\
\hline Insert point & \\
\hline
\end{tabular}

Fig. 16. Parameters for the creation of the distribution pattern of the 2D shape.

\section{Metodology for structural functionalisation}

This section presents an integral solution for the design of internal structures that can either be hollow or made from another material with a different density in order to modify cushioning properties and plantar pressure distribution of anatomical insoles and additions. This solution uses specific techniques that make it possible to manufacture the design by $3 \mathrm{D}$ printing based on FDM, avoiding the constraints of this type of manufacturing technique.

To carry out these processes, new CAD/CAE tools developed to systematise each of the process steps were employed. Apart from assisting the designer by structuring and automating the process steps, they help avoid errors and reduce the time needed to complete the process. These new resources were implemented and validated using IcadPAN $\AA$, the specific design software for insole design developed by the Spanish Footwear Technology Institute (INESCOP).

\subsection{Creation of functional structures}

A $3 \mathrm{D}$ insole generated by CAD software is defined by a series of surfaces joined together to form a closed object. These surfaces are: the bottom surface of the insole, which is in contact with the footwear sole; the upper surface of the insole, which is in contact with the foot; and the side surface, which corresponds to the insole profile and accounts for the insole thickness. Figure 10 shows all these surfaces separately. The tool herein described is intended to automate, according to certain parameters, the creation of one or more structures enclosed within said surfaces. For more clarity in the explanations, the insole is positioned in such a way that its longitudinal axis extending from the heel to the toe coincides with the $\mathrm{X}$ axis, and its rearmost point coincides with $\mathrm{X}=0.0 \mathrm{~mm}$. The insole is also centred in the $\mathrm{Y}$ axis in such a way that this axis divides the insole into two halves $\leqslant$ and the upper surface is oriented on the positive $\mathrm{Z}$ axis. $\leqslant$ Figure 10 also depicts the above-described position of the insole. With regard to the geometry of $3 \mathrm{D}$ additions, it is important to highlight that they do not have a side suface, but only bottom and upper surfaces. Figure 11 shows a 3D addition and the surfaces making it up separately.

The tool to create functional structures takes the surfaces defining the insole or addition geometry as a constraint inherent to its nature, in that the structures defined are to be parametrically distributed in the inside, without running through these external constraints. Furthermore, the user can establish other parameters to volumetrically define the area where the structures are to be created. The first parameter is the offset of the upper surface of the insole from the upper surface of the structures (see Figure 12a). Given that usually such structures remain hollow inside the insole, it is important to define enough offset for the layer deposited by FDM to be consistent and adequately finished. A longitudinal curve is used to define the bottom offset, which defines the lower level or ground of the structures (see Figure 12b). Normally this curve is parallel to the ground. However, in certain cases it may be necessary to define a 
variable bottom offset so that the structure properties can be altered according to their position and the desired specifications. The last parameter to define the creation zone is the start and end percentajes relative to the total insole length. This allows us to determine, for instance, whether the inside structure is to be located under the heel, under the metatarsal area or along the whole insole length. Figure 13 shows a general view of the tool interface for insole functionalisation, displaying the parameters that define the constraints for the creation of internal structures. Thanks to the parameters established for the creation zone, the designer does not need to create ad-hoc structures depending on the place where they are to be located, but it is the tool which automatically places them inside the insole in the desired position.

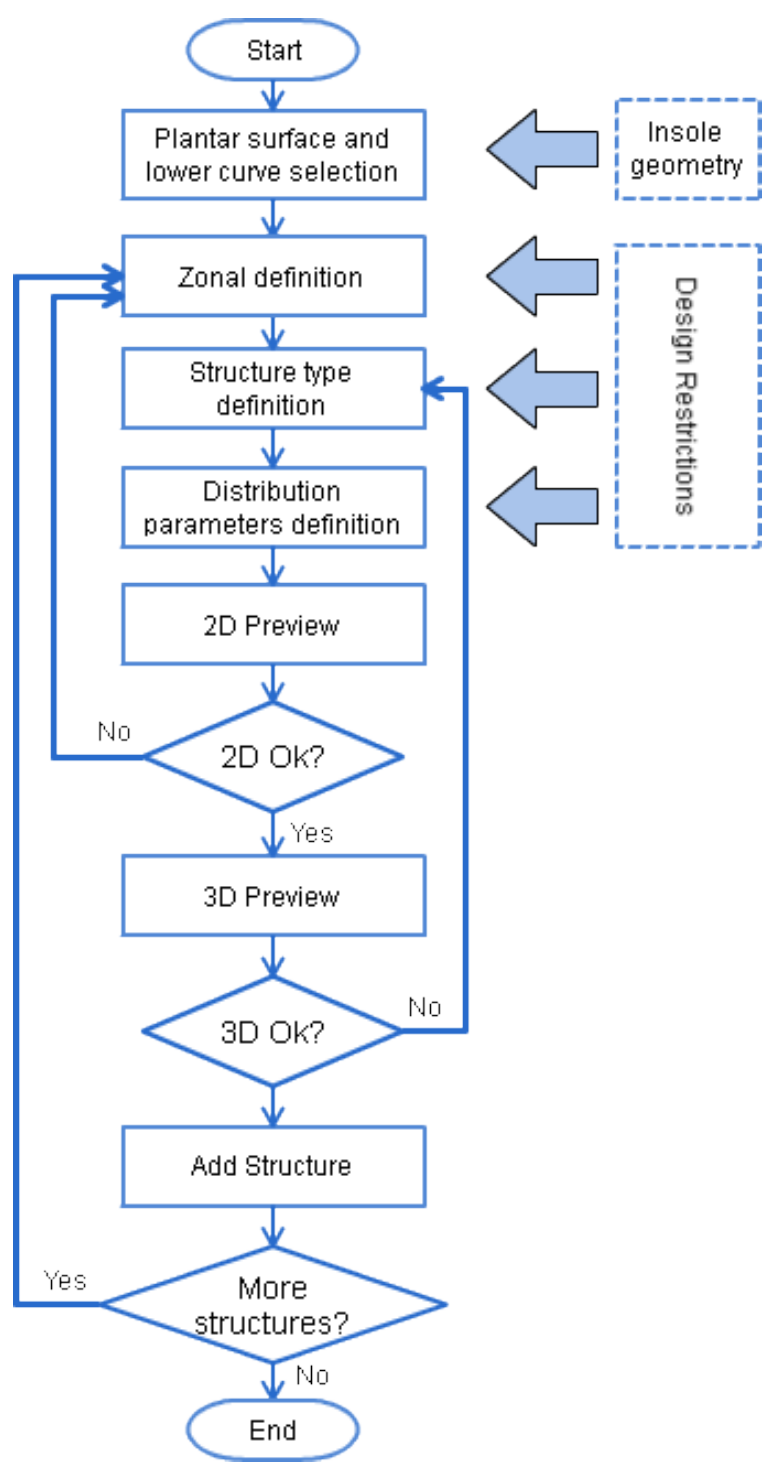

Fig. 17. Flow chart of functional structure creation process.

Functional internal structures are therefore created parametrically, being adapted to the geometry of the insole/addition and the functional requirements indicated by the user. Structures are made up from 3D patterns that are automatically replicated. Their creation process consists in: firstly, distributing the $2 \mathrm{D}$ geometric shape on the $\mathrm{Z}=0$ plane; then, from the lower level curve, two parallel curves in the $+\mathrm{Y}$ and $-\mathrm{Y}$ directions are created to generate a lower surface; then, the upper surface is copied and moved in the $-\mathrm{Z}$ direction as many millimetres as indicated by the defined offset, thus creating the upper surface; finally, the 2D curves are projected against the lower and upper surfaces. The resulting closed curve pairs constitute valid internal elements, disregarding the curves that do not have a corresponding curve or remain open. For each pair, the corresponding upper and lower surfaces are created. The side surface is obtained by geometrically extruding a curve against another. Figure 14 depicts the described algorithm. Figure 15 illustrates the $3 \mathrm{D}$ structures created from $2 \mathrm{D}$ shapes.

Input parameters for the creation of the distribution pattern provide flexibility to the designer when it comes to establishing the geometry of functional elements. Twodimensional geometric shapes from which the distribution for subsequent extrusion of internal surfaces is generated are as follows: (a) Rectangles, rhombus (diagonals) and circles, by means of which the relevant homonymous shapes are created. Offset (see figure 12c) indicates the distance from the profile curve of the upper surface. The number of structures is given by the Number of Rows and Number of Columns parameters. $V$ Separation and $H$ Separation are used to define width between the $\mathrm{X}$ and $\mathrm{Y}$ axes respectively. (b) Manual circles: these circle-type elements of variable diameter can be included by the designer independently in the desired position. (c) Pattern: by means of a line, a repetition pattern is created, which is longitudinally repeated along the insole or addition. The designer establishes the Offset from the profile curve of the upper surface. The element's Width is the distance on the $\mathrm{X}$ axis between its rear and front walls. Distance is the separation on the $\mathrm{X}$ axis between different structures. (d) Manual structure: the 2D pattern is manually designed and, based on this, the structure in the defined position is generated. Figure 16 shows the different creation modes for distribution patterns and the parameters defining each one.

Once the basis of this methodology has been exposed, the sequence that was established to create functional elements in insoles/additions is determined by a series of steps: 1) Upper surface selection. 2) Definition of the lower/ground curve. 3) Selection of the type of shape to be distributed. 4) Set up of distribution parameters and application area. 5) Obtaining of 2D pattern. In this stage the user can check in a fast way if the shape selected, its distribution pattern and application area are the appropriate ones. If not, distribution parameters will need to be changed. 6) Obtaining of the internal surfaces. In this stage, 3D structures are observable, and in the event that the result does not meet the requirements, it is also possible to redefine creation parameters. In the case that other structures are needed to be added in other areas of the insole, the user is to return to step 3. In Figure 17 the flow chart characterising this sequence is shown. 
Fig. 18. Automatic creation of the support. a) Original lower curve projected on the ground. b) Curve intersection and concatenation. c) Creation of support surfaces. d) Semitransparent view of the support added to the insole.
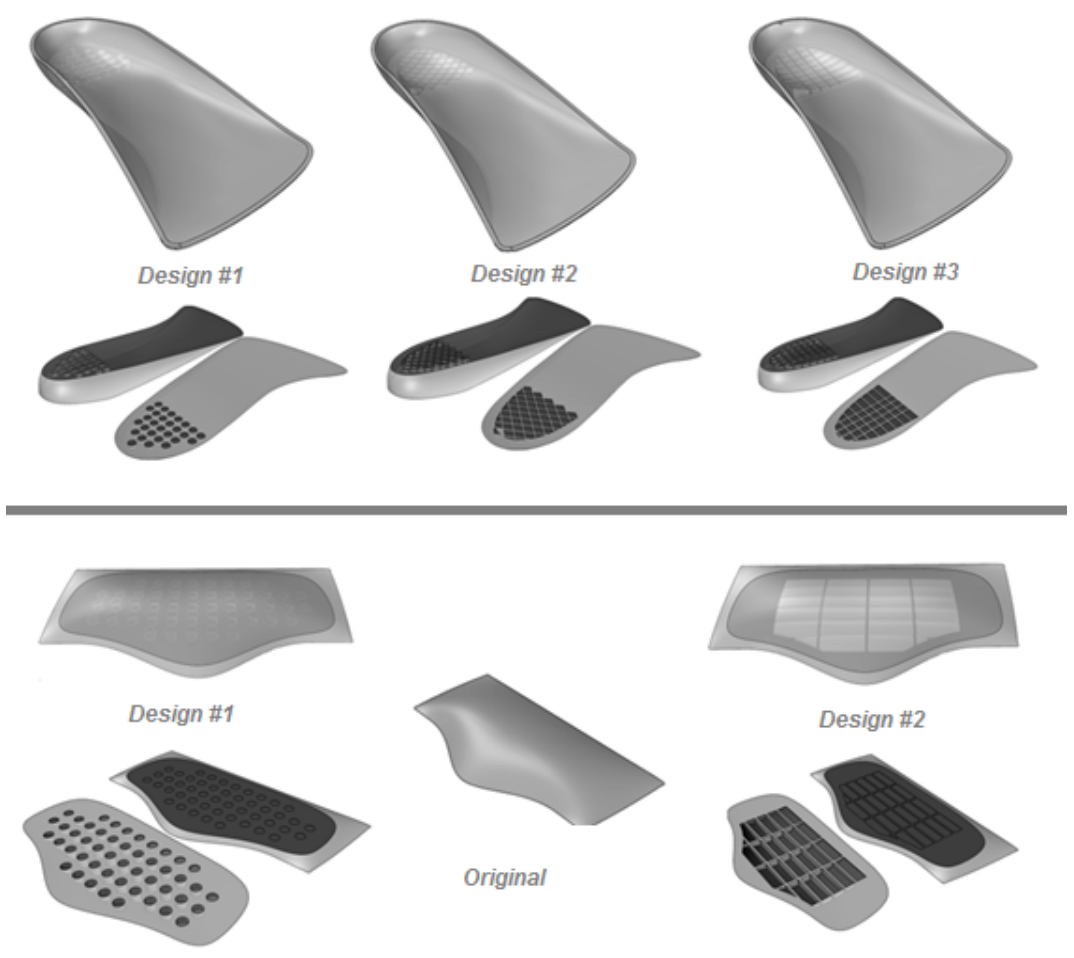

Original

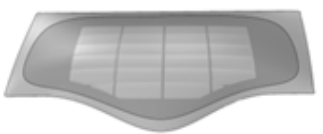

Design \#2



The main advantages of this methodology are: (a) High time consumption arising from handmade design of repetitive structures is drastically reduced. b) In the handmade process, designs are not reusable. In fact, the work required for modifying their shape, distribution, or location where they are intended to be applied is equal to that of creating them again from scratch. With this tool, it would be enough to modify simple parameters to change the element that is being distributed, the distribution pattern and and the application area of any internal structure. (c) Handmade design is subject to contain inaccuracies, either because of exceeding the boundaries of the insole, or because of the intersection of several internal surfaces. This would be avoided with a parametric tool based on a set of restrictions, such as the one described here. (d) Finally, this tool supports designer's decision making, in that it can help creating a quick series of variations upon an initial idea and decide accurately which one would best meet his/her requirements.

\subsection{Preparation for manufacturing by FDM}

One of the main limitations of low cost FDM-based printers is that the printing software processes geometry to be manufactured as a fully solid object, with no inside hollow, even though the shaped object in the CAD file does have it. This problem is directly linked to the methodology approached in this paper for the design and manufacture of functionalised insoles and addition, since the printing software by FDM will disregard structures contained in it that have been added. That is why this technique is presented, with which printing of an object with these features is feasible through a pre-processing of the geometry.

So that the 3D printing software can interpret internal structures, it would be necessary that they were not contained inside, but instead they belonged to the external surface of the object. This is achieved by separating the insole/addition into equal halves cut with a plane oriented in the $\mathrm{Z}$ axis, resulting in a top surface and a bottom surface. The bottom surface is in contact with the ground; it holds the lower half of internal structures and is sent to be manufactured in first place. The top surface is in contact with the foot and contains the upper half of internal structures. In order to be manufactured, material on the lower half is firstly extruded, so as to join both surfaces as a single object with no visible cut line. In Figure 18 an insole and an addition are shown 
containing different bottom structures and both underwent the described process.

This technique is developed automatically and in a transparent way for the user. The process performed is about calculating the average value of $Z$ position of gravity centre points of the side surface and each internal element. Then, a plane parallel to the ground in the $\mathrm{Z}$ position obtained is created. From the calculation of the geometric intersection of the plane with the mentioned elements, these are divided into two open halves. In order to obtain the closing surface, the cutting plane is cut with the external profile and the internal structures. Finally, the closing surface joins the lower half on one side and the upper half on the other, resulting in a pair of closed objects. This process is depicted in Figure 19.

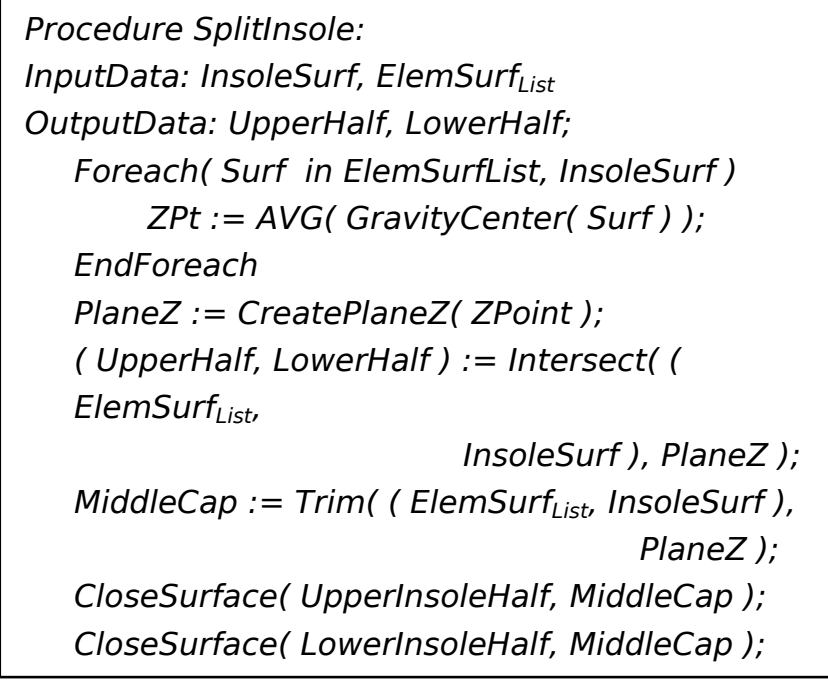

Fig. 19. Algorithm for the insole's separation in two halves.

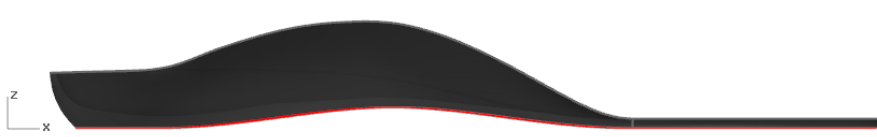

Fig. 20. Insole whose base has relief and is not in full contact with the ground.

\subsection{Support generation.}

In many cases, the bottom surface of the designed insole will be completely flat, and hence it would be in full contact with the ground. However, it may happen that the bottom part is given a certain relief and, therefore, the insole does not rest completely on $\mathrm{Z}=0$ plane. (see Figure 20). This may be due either to specific functional requirements or to geometry adaptation to a certain footwear sole.

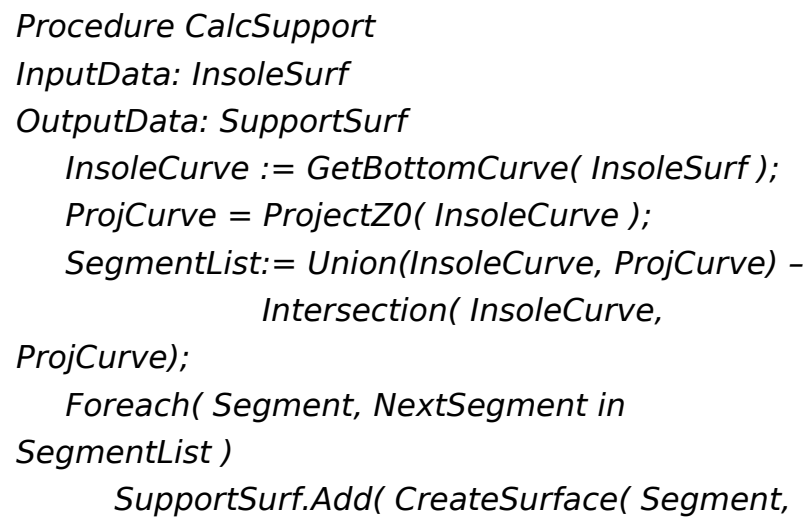

Fig. 21. Algorithm for generating the printing support.

Due to inherent constraints of 3D printers by FDM, the attempt to directly manufacture insoles with these features could fail, since in areas with no contact with the ground there is no base upon which material can be deposited. This situation may be solved by using double-head printers, with which it is possible to manufacture a support acting as scaffolding for the object. However, in practical terms, the printing support implies a further 3D object which has to be designed involving a time investment for the designer. In this section, a specifically developed procedure is presented by which the printing support for a $3 \mathrm{D}$ anatomical insole is automatically generated in case that the geometry of the bottom surface is not in full contact with the ground.

The process of automatic creation of printing support begins with obtaining the contour curve of the bottom surface. Afterwards, a plane oriented to $\mathrm{Z}$ axis of the coordinate system at a $0-\mathrm{mm}$ height is created. Then, the lower contour curve is projected upon this plane and one curve is intersected against the other. Bearing in mind that part of the lower contour is actually touching the ground, non-common segments are obtained from the intersections of both the projected curve and the lower curve of the insole. This four segments obtained are joint two to two and a surface is calculated from every pair, thus obtaining each surface: bottom, top and lateral of the support. The described algorithm is specified through Figure 21, whereas the different stages of this process are depicted in Figure 22. The generated support consists of a closed object whose base is in full contact to the ground, and therefore is suitable for FDM printing. 
Fig. 22. Automatic creation of the support. a) Original lower curve projected on the ground. b) Curve intersection and concatenation. c) Creation of support surfaces. d) Semitransparent view of the support added to the insole.
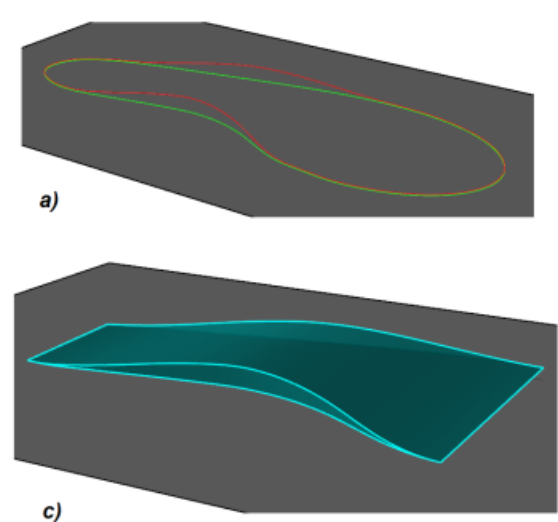

c)
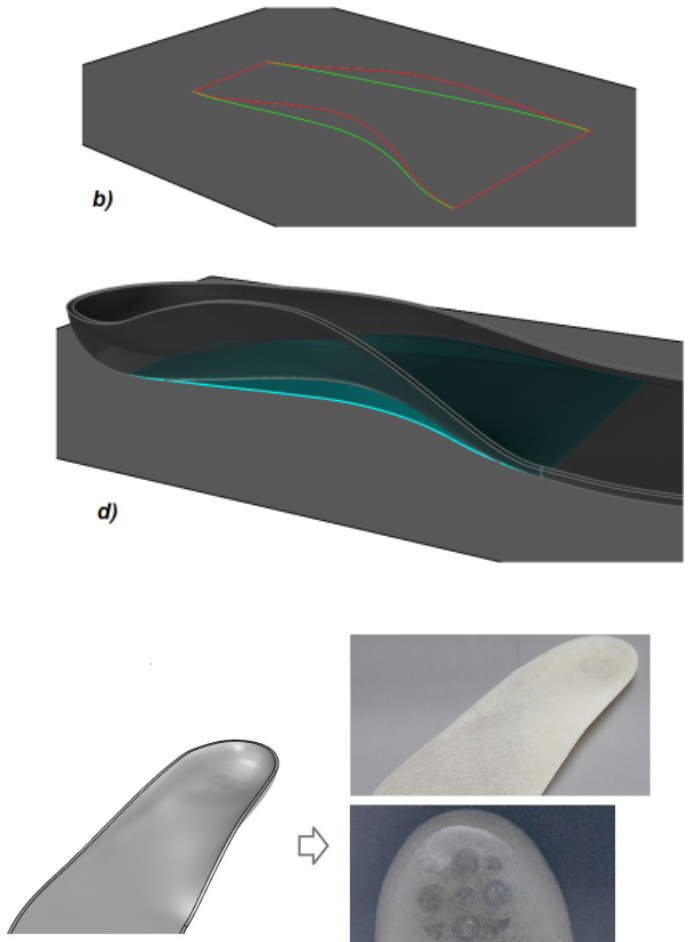

Insole 2

$\Rightarrow$
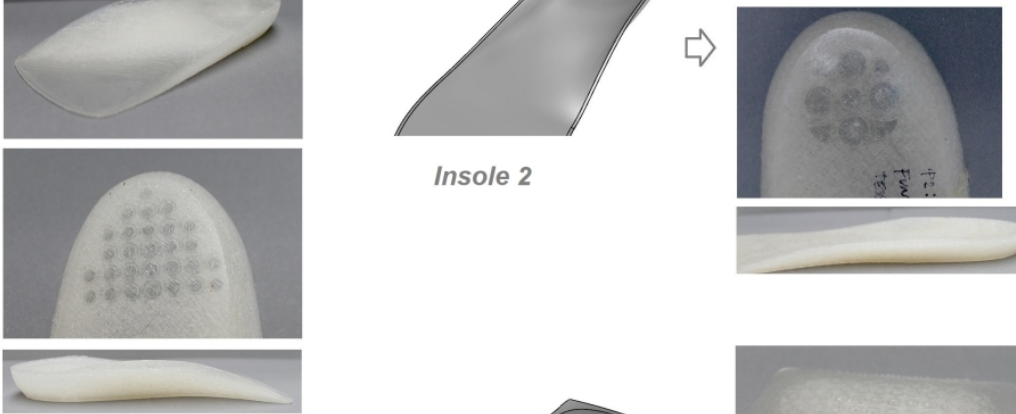

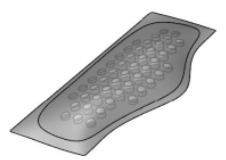

Addition



$\vec{\partial}$
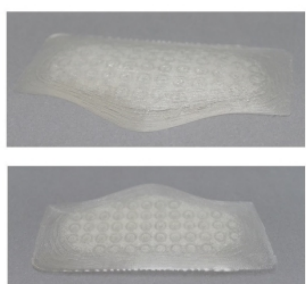

\section{Experimental}

The experimental stage was aimed to prove, firstly, that the developed techniques are suitable for FDM 3D printing, in that the element produced is correct and meets the requirements established in the design stage. Secondly, it was intended to prove that anatomical insoles and additions can be functionalised by adding internal structures intended to modify their shock absorption properties. For this purpose, a series of structures on different test pieces were designed and underwent several tests to analyse the shock absorption capacity of each one of them.
In the first validation stage, functional structures were added to several designs of insoles and additions using the CAD tool developed. They were subsequently manufactured by FDM using the techniques described herein (see Figure 23). Material employed was thermo-plastic polyurethane (TPU) filament. After visual inspection, it was concluded that the results obtained were correct, since internal structures showed a hollow geometry, as designed. The fact that the insoles and additions were divided into two halves prior to manufacturing did not affect the quality of the final result at all, since there was no discontinuance or cut line in the side surface. 


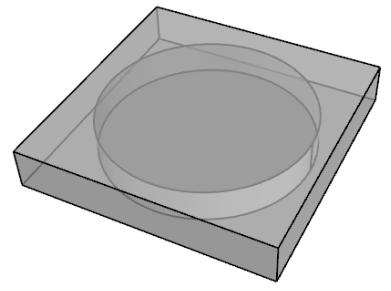

Test sample 1

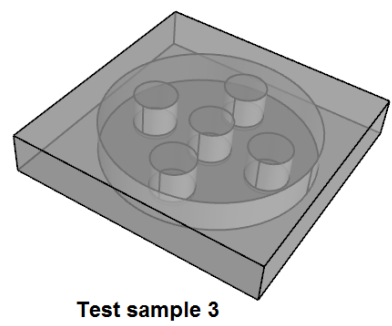



Test sample 2

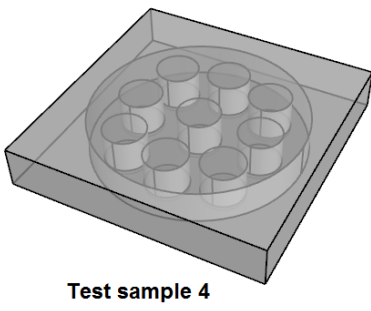

Test sample 4
Fig. 24. The four test pieces underwent a shock absorption test.
The second experimental stage consisted in checking how addition of internal structures modified shock absorption properties of the manufactured element. To this end, four different test pieces were designed and made from TPU as well. These consisted of one material block of

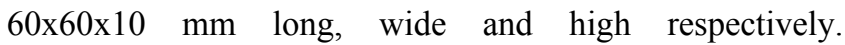
TestSample1 had an entirely-hollow inner cylinder with a radius of $25 \mathrm{~mm}$ and a height of $8 \mathrm{~mm}$ located in the centre of the block, acting as an air chamber. The rest of test pieces stemmed from TestSample1, but they had a certain number of cylinders or pivots of a radius of $5 \mathrm{~mm}$ acting as a skeleton. TestSample2 had only one material pivot located in the centre. TestSample3, apart from a central pivot, had four pivots located following the distribution of the corners of a square. Finally, TestSample4, besides the central pivot, had 8 pivots located following the distribution of an octagon on the test piece. Figure 24 illustrates the four test pieces designed.

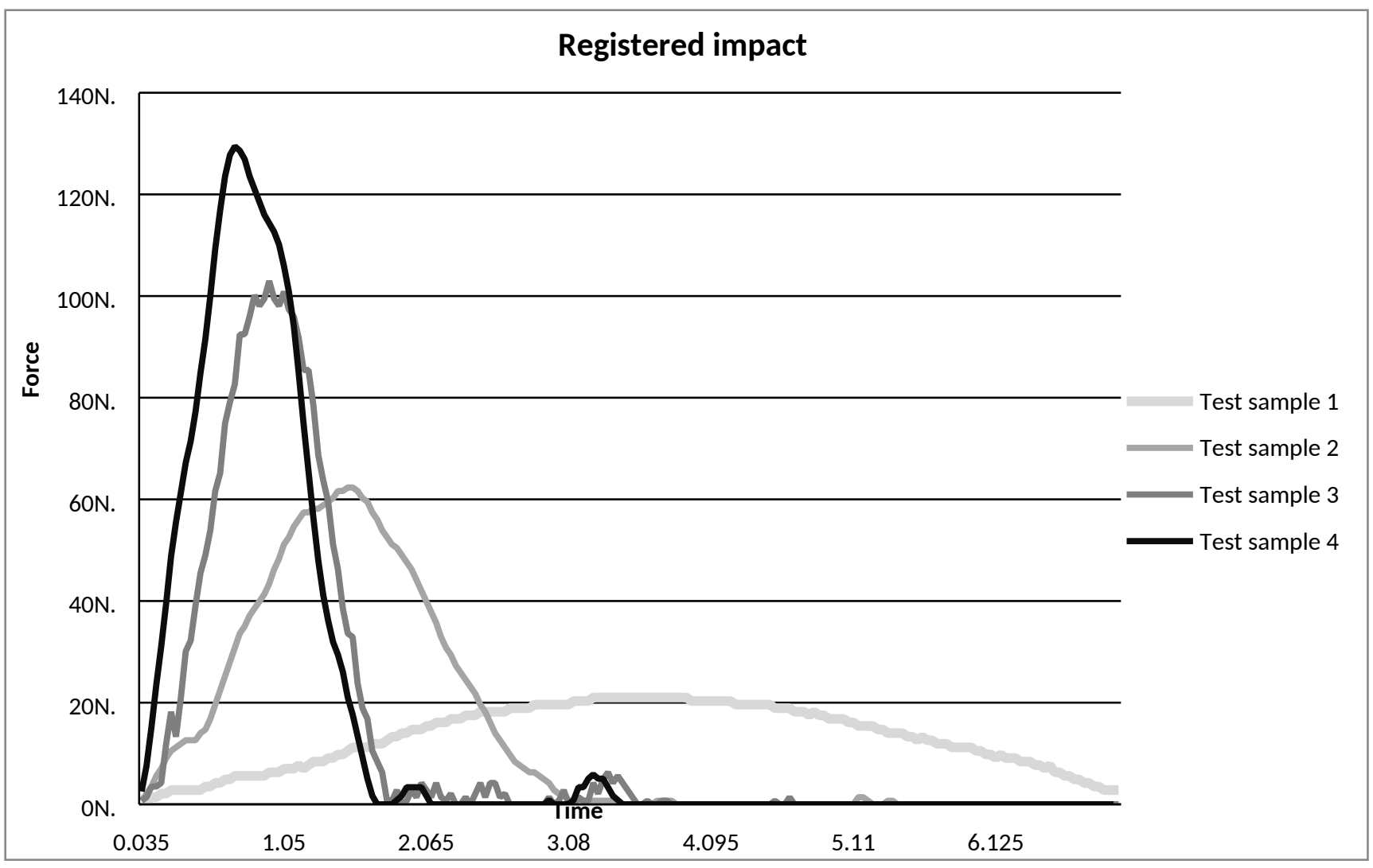

Fig. 25. Results from impact analysis. The impact force decreased as fewer pivots were contained inside the air chamber.

The test that the pieces underwent consisted in dropping a sphere of $28 \mathrm{~g}$. in mass and $16 \mathrm{~mm}$. in diameter from a height of $220 \mathrm{~mm}$. Using a load sensor, force (F) exerted by the sphere upon impact was obtained. This is how shock absorption capacity of the test pieces was compared, since the higher force the internal structure of the piece is able to absorb, the lower the force is exerted upon the sensor. The sensor generates an electrical signal (potential difference) in response to the force exerted upon it. By means of a previous calibration process, relationship between the potential difference recorded and the force was obtained, and therefore it was possible to transform electrical magnitudes into mechanical ones. 
The testing results are illustrated in Figure 25, which shows he impact force recorded upon the ball's falling on the different test pieces throughout the experiment duration. A graph showing a slight slope and a low force peak would mean a high level of shock absorption, since in that case the test piece would absorbs the force exerted by the ball when dropping, dissipating the impact over time. However, a steep slope and a high force peak would mean the opposite: the test piece would be able to absorb less force and the impact would be concentrated in a shorter period of time. According to TestSample1, which is entirely hollow, the maximum cushioning rate was obtained, having recorded a maximum force of 21.01 Newton. In the rest of the pieces, it was observed that the greater the number of pivots, the lower the cushioning properties. In TesSample 2 a maximum force of 61.34 N. was recorded, and in TestSample3, the maximum force recorded was 102.97. Finally, in TestSample4, a force of $129.45 \mathrm{~N}$. was recorded. The difference among the recorded forces in each test piece highlighted how it is possible to modify cushioning features of specific areas of the anatomical insole by using internal structures. Likewise, it was found that different types of structures provide different cushioning responses.

\section{Conclusions.}

Recent advances in additive manufacturing technologies give rise to new possibilities to produce objects. On the one hand, it is possible to incorporate additives in the constituent material of the product, so that it performs an additional function to that for which it was initially intended; on the other hand, this new technique allows the manufacture of objects with geometries that would not be feasible by traditional techniques, mainly due to the fact that the internal part of the object is never accessible, among other factors.

On the basis of the research performed and presented in this paper, it can be concluded that the footwear industry can benefit from the advantages that additive manufacturing brings about by applying it to the manufacture of anatomical insoles incorporating new functionalities. FDM-based 3D printers have become a suitable AM technology for use in the footwear industry thanks to their low cost. Moreover, as the insole is an element which is in direct contact with the foot, it may be convenient to add some antimicrobial agents to the manufacturing material to prevent proliferation of microorganisms. As far as geometry is concerned, it is possible to incorporate internal structures into the insole, which modify shock absorption properties.

An anatomical insole is, by definition, a customised element for a single user, but thanks to AM, it is plausible to achieve a higher level of customisation, conferring an added value on it thanks to its adaptation to particular cushioning needs of each user. This way, market trends are followed, where customers look for quality products that increasingly meet their requirements.

Nevertheless, the footwear industry lacks CAD tools to take full advantage of additive manufacturing in terms of design and manufacture of anatomical insoles. With the methodology described herein, implementation of functional structures is systematised through parametric design, reducing the time required for design and the possibility of making mistakes. Furthermore, the CAD model to be built is automatically processed in such a way that inherent constraints of FDM 3D printing are solved. This way of processing, although initially intended for insoles, can be applied as well to any object with these features. On the one hand, geometry is divided into two parts in a transparent manner in order to print its internal hollows, and hence overcome the problem that the printing software disregards these elements. On the other hand, a support is automatically generated, where necessary.

During the experimental stage, these methods for insole design and manufacture proved to be valid, achieving optimum results. In a second experiment, several test pieces containing different internal structures underwent shock absorption tests. The results revealed that the use and variability of their geometries favoured the modification of shock-absorption properties of the object. For future research, it is proposed to study the relationship between shock absorption capacity and geometry of internal structures (type of geometric shape, separation amongst elements, etc.) with the purpose of establishing a procedure by which structures are built up automatically on the sole basis of the shock absorption coefficient envisaged. 


\section{References}

[1] Riegger CL (1988) Anatomy of the ankle and foot. Phys Ther. 68(12):1802-14.

[2] Buldt A, Levinger P, Murley G, Menz H, Nester C, Landorf K (2015) Foot posture is associated with kinematics of the foot during gait: A comparison of normal, planus and cavus feet. Gait Posture. ;42(1):42-8

[3] Brodsky J, Kourosh S, Stills M, Mooney V (1988) Objective Evaluation of Insert Material for Diabetic and Athletic Footwear. Foot Ankle. 9(3):111-6.

[4] Huang S, Liu P, Mokasdar A, Hou L (2003) Additive manufacturing and its societal impact: a literature review. Int J Adv Manuf Technol 67(5):1191-1203

[5] ISO/ASTM 52900:2015 Additive manufacturing. General principles. Terminology

[6] Fages-Santana E (2013) Research on nanosilver addition to polypropylene fibers in order to obtain bioactive textile materials suitable for medical industry. Doctoral dissertation.

[7] Yang S, Zhao YF (2015). Additive manufacturing-enabled design theory and methodology: a critical review. The International Journal of Advanced Manufacturing Technology 80(1-4):327342.

[8] Cacace LA, Hillstrom H, Dufour AB, Hannan MT (2013) The association between pes planus foot type and the prevalence of foot disorders: the Framingham foot study. Osteoarthritis and Cartilage 21:S166-S167

[9] Fernández-Seguín LM, Diaz Mancha JA, Sánchez Rodríguez R, Escamilla Martínez E, Gómez Martín B, Ramos Ortega J. (2013) Comparison of plantar pressures and contact area between normal and cavus foot. Gait Posture. 39(2):789-92

[10] Chen WP1, Ju CW, Tang FT. (2003) Effects of total contact insoles on the plantar stress redistribution: a finite element analysis. Clin Biomech 18(6):S17-24

[11] Leber C, Evanski PM (1986) A comparison of shoe insole materials in plantar pressure relief Prosthet Orthot Int. 10(3):1358 .

[12] García AC1, Durá JV, Ramiro J, Hoyos JV, Vera P. (1994) Dynamic Study of Insole Materials Simulating Real Loads. Foot Ankle Int. ;15(6):311-23

[13] Shimazaki Y, Nozu S, Inoue T (2016) Shock-absorption properties of functionally graded EVA laminates for footwear design. Polymer Testing 54:98-103

[14] Brodsky, J W, Pollo F E, Cheleuitte D, \& Baum B S (2007). Physical properties, durability, and energy-dissipation function of dual-density orthotic materials used in insoles for diabetic patients. Foot \& ankle international, 28(8), 880-889.

[15] Novakova-Marcincinova L, Novak-Marcincin J, Barna J, Torok J (2012). Special materials used in FDM rapid prototyping technology application. IEEE 16th International Conference on Intelligent Engineering Systems (INES) (pp. 73-76)

[16] Giordano RA, Wu BM, Borland SW, Cima LG, Sachs EM, Cima MJ (1997) Mechanical properties of dense polylactic acid structures fabricated by three dimensional printing. Journal of Biomaterials Science, Polymer Edition, 8(1), 63-75.

[17] Weng Z, Wang J, Senthil T, Wu L (2016) Mechanical and thermal properties of $\mathrm{ABS} /$ montmorillonite nanocomposites for fused deposition modeling 3D printing. Materials \& Design, 102, 276283.

[18] Yue J, Zhao P, Gerasimov J Y, van de Lagemaat M, Grotenhuis A, Rustema-Abbing M, Ren Y (2015) 3D-Printable Antimicrobial Composite Resins. Advanced Functional Materials, 25(43), 67566767.

[19] Alhijjaj M, Belton P, Qi S (2016) An investigation into the use of polymer blends to improve the printability of and regulate drug release from pharmaceutical solid dispersions prepared via fused deposition modeling (FDM) 3D printing. European Journal of Pharmaceutics and Biopharmaceutics, 108, 111-125.

[20] Gibson I, Rosen DW, Stucker B (2010) Additive Manufacturing technologies: rapid prototyping to direct digital manufacturing. Springer, US http://www.springer.com/us/book/9781493921126

[21] Sisca FG, Angioletti CM, Taisch M, Colwill JA (2016). Additive manufacturing as a strategic tool for industrial competition. In Research and Technologies for Society and Industry Leveraging a better tomorrow (RTSI), 2016 IEEE 2nd International Forum on (pp. 1-7). IEEE.

[22] Vayre B, Vignat F, Villeneuve F, (2012) Designing for Additive Manufacturing Procedia CIRP 3:632-637

[23] Minetola P, Iuliano L, Marchiandi G (2016). Benchmarking of FDM machines through part quality using IT grades. Procedia CIRP, 41:1027-1032.

[24] Davia-Aracil M, Jimeno-Morenilla A, Salas F (2016). A new methodological approach for shoe sole design and validation. The International Journal of Advanced Manufacturing Technology, 86(9):3495-3516

[25] Aristides AG, Requicha J, Rossignac R (1992) Solid modelling and beyond. IEEE Comput Graph Appl 12(5):31-44 